\title{
Anwendung dynamisch erweiterter Beobachter zur robusten
} Fehlerisolation

\author{
Application of Dynamically Extended Observers to Robust Fault Isolation
}

\author{
Arne Wahrburg, Jürgen Adamy, Technische Universität Darmstadt
}

\begin{abstract}
Zusammenfassung Dieser Beitrag befasst sich mit dem Entwurf von dynamisch erweiterten Fehlerisolationsbeobachtern (FIOs). Dazu wird zunächst eine Methode zum Entwurf dynamischer Entkopplungsregler vorschlagen. Basierend auf der Dualität von Entkopplungsregelungen und Fehlerisolationsbeobachtern lässt sich hieraus ein Verfahren zum Entwurf von dynamischen Fehlerisolationsbeobachtern ableiten, welches gegenüber klassischen FIOs zusätzliche Freiheitsgrade bietet. Diese werden in diesem Beitrag dazu genutzt, die Robustheit bezüglich hochfrequenter Stö-
\end{abstract}

rungen zu verbessern. $\leadsto$ Summary This paper presents a method to design dynamically extended fault isolation observers (FIOs). Therefore, a design procedure for dynamic non-interacting controllers is proposed. Based on the duality between non-interacting control and observerbased fault isolation a technique to design dynamic FIOs is deduced, which provides additional degrees of freedom compared to static FlOs. These are employed in this article to increase robustness with respect to high-frequency disturbances.

Schlagwörter Dynamisch erweiterte Beobachter, Fehlerisolationsbeobachter, robuste Fehlerisolation, Entkopplungsregelung $\leadsto$ Keywords Dynamically extended observers, fault isolation observers, robust fault isolation, non-interacting control

\section{Einleitung}

Die hohe Komplexität technischer Systeme und steigende Anforderungen an die Zuverlässigkeit bei gleichzeitigem Kostendruck begründen das stete Interesse an modellbasierten Fehlerdiagnoseverfahren in den vergangenen Jahren und Jahrzehnten. Neben identifikationsbasierten Ansätzen und Diagnosemethoden mit Paritätsgleichungen [13] befassen sich zahlreiche Arbeiten mit der beobachterbasierten Detektion und Isolation von Fehlern (s. z. B. [2;9] für einen Überblick).

In praktischen Anwendungen unterliegen Systeme immer Störungen sowie gewissen parametrischen Unsicherheiten. Diese können u.a. durch externe Einflüsse hervorgerufen werden oder auch durch intrinsische Effekte, wie z. B. unmodellierte Dynamiken. Aus die- sem Grund ist eines der wesentlichen Probleme aller modellbasierten Fehlerdiagnoseverfahren die Robustheit bezüglich solcher Störungen und Unsicherheiten. Ziel ist es, das Diagnosesystem möglichst sensitiv auf zu erkennende Fehler zu machen und gleichzeitig möglichst unempfindlich gegenüber Störungen und Unsicherheiten.

Im Bereich der Fehlerdetektion stehen üblicherweise zahlreiche Freiheitsgrade zur Verfügung, um die Robustheit zu steigern (s.z.B. [21;37]). In jüngerer Vergangenheit haben sich zahlreiche Arbeiten mit der Fehlerdetektion in beschränkten Frequenzbereichen befasst (s. z. B. [6;16;32-34]), die auf dem verallgemeinerten Kalman-Yakubovich-Popov Lemma basieren [14]. Dadurch lässt sich Wissen über die relevanten Frequenz- 
bereiche von Fehlern und Störungen in den Entwurf einbringen. Während Fehler häufig niederfrequent sind (z.B. konstante Offsets), treten oftmals hochfrequente Störungen auf (z. B. Sensorrauschen).

Wird dagegen eine Aussage über Art und Ort verschiedener, eventuell gleichzeitig auftretender Fehler benötigt, spricht man von Fehlerisolation. Ein klassischer Ansatz dazu beruht auf der Implementierung von mehreren, spezifisch ausgelegten Fehlerdetektionsbeobachtern, so genannten Beobachterbänken [11]. Im Gegensatz dazu ermöglichen Fehlerisolationsbeobachter (FIOs) die Isolation von Fehlern mittels eines einzigen Beobachters [18]. Die Grundidee der FIOs beruht auf der Dualität zu Entkopplungsregelungen. Dadurch lassen sich die Resultate auf diesem Gebiet, zurückgehend auf [10], im Bereich der Fehlerisolation anwenden. In diesem Fall wird jedoch ein Großteil der zur Verfügung stehenden Freiheitsgrade zur Gewährleistung der Isolationseigenschaft des Beobachters benötigt, sodass eine adäquate Robustheit schwierig sicherzustellen ist. Lediglich im Fall zusätzlicher Sensorik, welche weitere Freiheitsgrade bedingt, liegen bisher Ergebnisse vor, die sich diesem Thema widmen (s. z. B. $[1 ; 15 ; 17])$.

In diesem Beitrag wird ein neuer Ansatz vorgestellt, welcher ohne solche zusätzliche Sensorik weitere Freiheitsgrade im Entwurf bereitstellt. Er stützt sich auf dynamisch erweiterte Beobachter, die in [24] vorgestellt wurden. Diese wurden in [7] zur Platzierung von Nullstellen eingesetzt, um bestimmte Störfrequenzen in der Fehlerdetektion zu blockieren. Weiterhin ist in [36] gezeigt, wie sich eine gemischte $H_{-} / H_{\infty}$-Fehlerdetektion mithilfe von dynamisch erweiterten Beobachtern realisieren lässt. Darüber hinaus finden sich Anwendungen im Bereich der Detektion von Sensorfehlern in nichtlinearen Systemen [26] und der simultanen Regelung und Fehlererkennung [8].

In diesem Artikel wird erörtert, wie sich dynamisch erweiterte Beobachter auch in der Fehlerisolation einsetzen lassen. Dazu wird in Abschnitt 3 zunächst die Dualität zwischen Entkopplungsregelungen und Fehler-isolationsbeobachtern herausgearbeitet. Ausgehend von diesen Überlegungen wird in Abschnitt 4 ein Verfahren vorgestellt, welches den Entwurf von dynamischen Entkopplungsregelungen erlaubt. Im Gegensatz zu klassischen Entkopplungsregelungen ergeben sich daraus zusätzliche Freiheitsgrade, indem die Ordnung der Diagonalelemente der Übertragungsmatrix des geschlossenen Kreises erhöht wird. Aufgrund der Dualität lässt sich daraus unmittelbar der Entwurf dynamisch erweiterter Fehlerisolationsbeobachter ableiten (s. Abschnitt 5). Hier wird gezeigt, wie sich die zusätzlichen Freiheitsgrade zur Steigerung der Robustheit bezüglich hochfrequenter Störungen nutzen lassen, was auch anhand eines Simulationsbeispiels in Abschnitt 6 demonstriert wird. Weitere Anwendungen können im Rahmen dieses Beitrages nur angedeutet werden.

\section{Problemstellung}

Betrachtet werden lineare zeitinvariante Mehrgrößensysteme der Form

$\dot{\boldsymbol{x}}=A \boldsymbol{x}+B \boldsymbol{u}+\boldsymbol{E}_{a} f+B_{d} d$,

$y=C x+D_{d} d$

mit $\boldsymbol{x} \in \mathbb{R}^{n}, \boldsymbol{u} \in \mathbb{R}^{n_{u}}, \boldsymbol{f} \in \mathbb{R}^{n_{f}}, \boldsymbol{d} \in \mathbb{R}^{n_{d}}, \boldsymbol{y} \in \mathbb{R}^{n_{y}}$ und Matrizen entsprechender Dimensionen. Behandelt werden in diesem Beitrag quadratische Systeme, d. h. es gilt $n_{y}=n_{f}$. Dabei bezeichnet $f$ die auf das System einwirkenden Fehler, die es zu detektieren und isolieren gilt. Während in [9] ein Verfahren beschrieben wird, welches die direkte Berücksichtigung von Sensorfehlern erlaubt, werden diese in (1b) nicht explizit berücksichtigt, da sie nach [23] als Pseudo-Aktorfehler umformuliert werden können. Im Vektor $\boldsymbol{d}$ werden Störungen zusammengefasst, deren Einfluss auf die Fehlerisolation möglichst unterdrückt werden soll. Die Störungen können sowohl Mess- und Systemrauschen als auch parametrische Unsicherheiten in den Systemmatrizen umfassen, wenn diese als $\boldsymbol{d}=\Delta \boldsymbol{A} \boldsymbol{x}+\Delta \boldsymbol{B} \boldsymbol{u}$ interpretiert werden.

Mithilfe eines Residuengenerators werden nun Residuen $r \in \mathbb{R}^{n_{f}}$ erzeugt. Beim Einsatz klassischer Beobachter erfolgt dies mittels

$\dot{\hat{x}}=A \hat{x}+B u+L(y-C \hat{x})$,

$r=V(y-C \hat{x})$,

woraus sich mit der Substitution $\boldsymbol{e}=\boldsymbol{x}-\hat{\boldsymbol{x}}$ die Übertragungsmatrix zwischen Fehlern und Residuen zu

$\boldsymbol{G}_{r f}(s)=V C\left(s \boldsymbol{I}_{n}-(A-L C)\right)^{-1} \boldsymbol{E}_{a}$

ergibt. Offensichtlich ist eine Isolation der einzelnen Fehler möglich, wenn $\boldsymbol{G}_{r f}(s)$ Diagonalgestalt hat, d. h.

$\boldsymbol{G}_{r f}(s)=\operatorname{diag}\left(g_{11}(s), \ldots, g_{n_{f} n_{f}}(s)\right)$,

da dann jedes Residuum sensitiv bezüglich genau eines Fehlers ist. Somit kann im eingeschwungenen, störungsfreien Fall von den Residuen eindeutig auf die Fehler zurückgeschlossen werden.

Alternativ zu klassischen Beobachtern der Form (2) werden in dieser Arbeit dynamisch erweiterte Beobachter herangezogen. Diese verwenden keine statische Rückführung der Differenz zwischen Strecken- und Beobachterausgang, sondern eine dynamische. Obwohl es sich auch beim Luenberger-Beobachter um ein dynamisches System handelt, wird dieser aus dem genannten Grund vereinfachend als statischer Beobachter bezeichnet, im Gegensatz zu dynamischen Beobachtern der Form

$$
\begin{aligned}
& \dot{\hat{x}}=A \hat{x}+B u+v, \\
& v=L_{i} z+L_{p}(y-C \hat{x}), \\
& \dot{z}=\Phi z+\Gamma(y-C \hat{x}) .
\end{aligned}
$$


Diese Struktur wurde in [24] vorgestellt und verfügt wegen $z \in \mathbb{R}^{v}$ über eine interne Beobachterdynamik. Zur Residuengenerierung wird im vorliegenden Beitrag

$r=V_{1}(y-C \hat{x})+V_{2} z$

herangezogen. Ein dynamischer Beobachter (5) kann als Zustandsraumrealisierung eines allgemeinen linearen zeitinvarianten Residuengenerators [9] der Form

$\boldsymbol{r}(s)=\boldsymbol{R}(s)(\boldsymbol{y}(s)-\hat{y}(s))$

aufgefasst werden. Dabei ist $\hat{\boldsymbol{y}}(s)$ der geschätzte Ausgang eines vollständigen Beobachters der Form (2a) und $\boldsymbol{R}(s)$ ein stabiles Postfilter. In Abschnitt 5.1 wird näher auf den Zusammenhang eingegangen.

Die Übertragungsmatrix zwischen Fehlern und Residuen ergibt sich bei Verwendung dynamischer Beobachter $\mathrm{zu}$

$\boldsymbol{G}_{r f}(s)=C_{r f}\left(s \boldsymbol{I}_{n+v}-A_{r f}\right)^{-1} \boldsymbol{B}_{r f}+\boldsymbol{D}_{r f}$

mit

$$
\begin{gathered}
A_{r f}=\left[\begin{array}{cc}
A-L_{p} C & -L_{i} \\
\Gamma C & \Phi
\end{array}\right], B_{r f}=\left[\begin{array}{c}
E_{a} \\
0
\end{array}\right], \\
C_{r f}=\left[\begin{array}{ll}
V_{1} C & V_{2}
\end{array}\right], \quad D_{r f}=0 .
\end{gathered}
$$

Ziel ist es, die Matrizen $\boldsymbol{L}_{p}, \boldsymbol{L}_{i}, \boldsymbol{\Phi}, \boldsymbol{\Gamma}, \boldsymbol{V}_{1}$ und $\boldsymbol{V}_{2}$ so zu wählen, dass (4) ebenfalls erfüllt ist und dabei eine ausreichend hohe Erkennungsgeschwindigkeit von Fehlern sowie eine möglichst gute Unterdrückung hochfrequenter Störungen erreicht wird.

Anmerkung 1. Wie in der Einleitung erwähnt, kann eine Fehlerisolation auch mittels geeignet ausgelegter Beobachterbänke erfolgen [11]. Dazu wird jeweils ein Beobachter so parametriert, dass das generierte Residuum lediglich von einem ihm zugeordneten Fehler beeinflusst wird [9]. Eine solche Beobachterbank lässt sich ebenfalls als dynamischer Beobachter der Form (5) darstellen. Ziel dieses Beitrages ist es jedoch, die Fehlerisolation mittels eines einzigen geeignet parametrierten Beobachters zu bewerkstelligen und damit den Implementierungsaufwand des Diagnosesystems gering zu halten.

\section{Dualität zwischen Entkopplungsregelungen und Fehlerisolationsbeobachtern}

Ein System der Form (1) mit $\boldsymbol{f}=\mathbf{0}, \boldsymbol{d}=\mathbf{0}$ wird durch $(\boldsymbol{A}, \boldsymbol{B}, \boldsymbol{C})$ abgekürzt. Wird es mittels einer statischen linearen Zustandsrückführung $\boldsymbol{u}=-\boldsymbol{K} \boldsymbol{x}+\boldsymbol{F} \boldsymbol{w}$ geregelt, so erhält man als Übertragungsmatrix zwischen Führungsgrößen $w$ und Ausgangsgrößen $y$

$\boldsymbol{G}_{\boldsymbol{y} \boldsymbol{w}}(s)=\boldsymbol{C}\left(s \boldsymbol{I}_{n}-(\boldsymbol{A}-\boldsymbol{B} \boldsymbol{K})\right)^{-1} \boldsymbol{B} \boldsymbol{F}$.

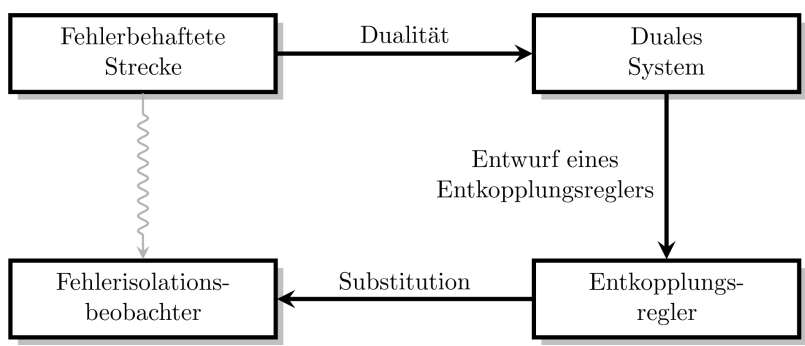

Bild 1 Dualitätsbasierter FIO-Entwurf.

Ziel einer Entkopplungsregelung [10] ist es, der Matrix $\boldsymbol{G}_{\boldsymbol{y} w}(s)$ Diagonalgestalt aufzuprägen. Dann gilt $\boldsymbol{G}_{\boldsymbol{y} \boldsymbol{w}}(s)=$ $G_{y w}^{\top}(s)$ und somit

$\boldsymbol{G}_{\boldsymbol{y} w}(s)=\boldsymbol{F}^{\top} \boldsymbol{B}^{\top}\left(s \boldsymbol{I}_{n}-\left(\boldsymbol{A}^{\top}-\boldsymbol{K}^{\top} \boldsymbol{B}^{\top}\right)\right)^{-1} \boldsymbol{C}^{\top}$.

Vergleicht man nun (10) mit (3), so wird deutlich, dass es sich beim Entwurf von Entkopplungsregelungen und Fehlerisolationsbeobachtern um duale Probleme handelt. Nach Bild 1 kann man Fehlerisolationsbeobachter folglich durch den Entwurf von Entkopplungsreglern für das duale System parametrieren. Diese Erkenntnis motiviert die folgende Annahme bezüglich (1), welche in Abschnitt 4 näher erörtert wird.

Annahme 1. Das quadratische System $\left(\boldsymbol{A}^{\top}, \boldsymbol{C}^{\top}, \boldsymbol{E}_{a}^{\top}\right)$ ist statisch stabil entkoppelbar.

Ist sie erfüllt, so wird das System $\left(\boldsymbol{A}, \boldsymbol{E}_{a}, \boldsymbol{C}\right)$ als statisch stabil fehlerisolierbar bezeichnet.

\section{Dynamische Entkopplungsregler}

Entsprechend den Ausführungen in Abschnitt 3 lässt sich auch der Entwurf dynamischer Fehlerisolationsbeobachter auf dynamische Entkopplungsregler zurückführen. In diesem Abschnitt wird ein neues Verfahren zum Entwurf solcher dynamischer Regler für quadratische, statisch stabil entkoppelbare Systeme vorgestellt.

Dabei werden vollständig steuerbare Systeme $(\boldsymbol{A}, \boldsymbol{B}, \boldsymbol{C})$ betrachtet, für die $n_{u}=n_{y}$ gilt. Die Differenzenordnungen $\delta_{i}$ der jeweiligen Ausgänge sind definiert durch

$\delta_{i}=\min _{k}\left\{k \geq 1: \boldsymbol{c}_{i}^{\top} \boldsymbol{A}^{k-1} \boldsymbol{B} \neq \mathbf{0}^{\top}\right\}$,

wobei $\boldsymbol{c}_{i}^{\top}$ die $\boldsymbol{i}$-te Zeile von $\boldsymbol{C}$ darstellt. Auf Grundlage der Differenzenordnungen berechnet sich die Entkoppelbarkeitsmatrix $\mathrm{zu}$

$D^{*}=\left[\begin{array}{c}\boldsymbol{c}_{1}^{\top} \boldsymbol{A}^{\delta_{1}-1} \boldsymbol{B} \\ \vdots \\ \boldsymbol{c}_{n_{y}}^{\top} A^{\delta_{n_{y}-1}} \boldsymbol{B}\end{array}\right]$.

Es lässt sich zeigen, dass die Regularität von $D^{*}$ notwendig und hinreichend für die Existenz eines statischen Entkopplungsreglers ist, und dass Minimalphasigkeit von $(\boldsymbol{A}, \boldsymbol{B}, \boldsymbol{C})$ dann die stabile Entkoppelbarkeit garantiert (s. z. B. [27]). Dementsprechend darf das $\operatorname{System}(\boldsymbol{A}, \boldsymbol{B}, \boldsymbol{C})$ 


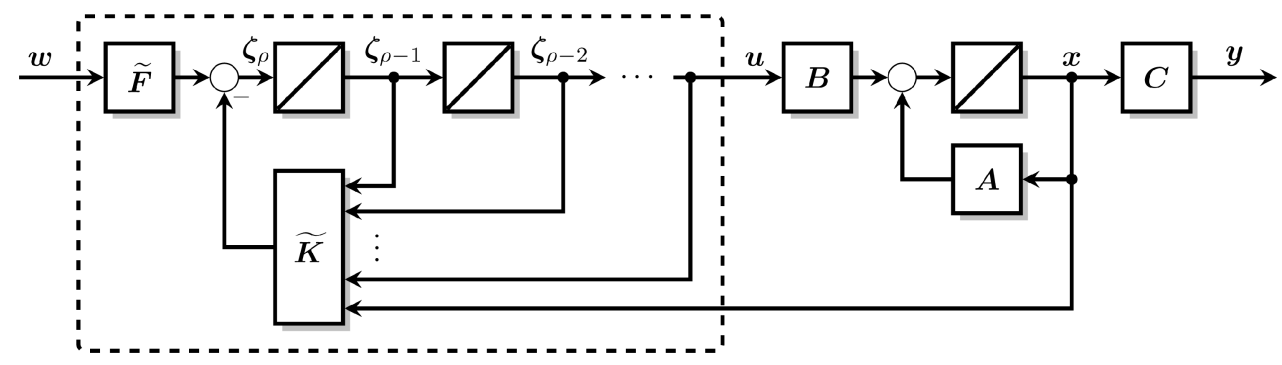

Bild 2 Dynamischer Entkopplungsregler.

keine invarianten Nullstellen in der geschlossenen rechten $s$-Halbebene besitzen. Der Entwurf kann dann z. B. nach [10] oder [27] erfolgen. Für den in diesem Beitrag nicht behandelten Fall $n_{u}>n_{y}$ (auch bekannt als Morgan's Problem) existieren nach gegenwärtigem Stand der Technik noch keine allgemeingültigen notwendigen und hinreichenden Bedingungen für die statische Entkoppelbarkeit von linearen Systemen. Die Resultate beschränken sich jeweils auf eingeschränkte Systemklassen (s. z. B. $[12 ; 28 ; 35]$ ).

Im Allgemeinen werden dynamische Entkopplungsregler eingesetzt, wenn ein System entweder aufgrund der Singularität von $D^{*}$ oder der Nicht-Minimalphasigkeit von $(\boldsymbol{A}, \boldsymbol{B}, \boldsymbol{C})$ nicht statisch stabil entkoppelbar ist. Neben dem algebraischen Ansatz in [3] und Frequenzbereichslösungen wie in $[4 ; 5]$ und $[22]$ existieren auch Lösungen im Zustandsraum [19]. Im Folgenden wird jedoch entsprechend Annahme 1 stets die statisch stabile Entkoppelbarkeit von $(\boldsymbol{A}, \boldsymbol{B}, \boldsymbol{C})$ vorausgesetzt. Der hier vorgestellte Entwurf eines dynamischen Reglers ist daher für die Entkopplung nicht notwendig. Vorteile ergeben sich jedoch wie in Abschnitt 5.1 gezeigt wird beim dualitätsbasierten Entwurf von Fehlerisolationsbeobachtern.

Wie in Abschnitt 3 erläutert haben Entkopplungsregler zum Ziel, der Matrix $\boldsymbol{G}_{\boldsymbol{y} w}(s)$ Diagonalgestalt aufzuprägen. Klassischerweise wird dabei nach [10] für die Diagonalelemente

$g_{i i}(s)=\frac{p_{i 0}}{s^{\delta_{i}}+\cdots+q_{i 1} s+q_{i 0}}$

angesetzt. Hier soll nun die Ordnung der Nennerpolynome in den einzelnen Übertragungspfaden um einen frei wählbaren Parameter $\rho$ erhöht werden, was sich durch

$g_{i i}(s)=\frac{p_{i 0}}{s^{\delta_{i}+\rho}+q_{i \delta_{i}+\rho-1} s^{\delta_{i}+\rho-1}+\ldots+q_{i 1} s+q_{i 0}}$

beschreiben lässt. Ein solches Übertragungsverhalten lässt sich erzielen, indem vor die Strecke eine Kette von $\rho$ Integratorblöcken geschaltet wird und für diese erweiterte Strecke ein klassischer Entkopplungsregler entworfen wird (s. Bild 2), was im Folgenden gezeigt wird.

Der Ausgang des letzten Integratorblocks ist die Stellgröße $\boldsymbol{u}$, die Ausgänge der vorgeschalteten Integratorblöcke werden mit $\zeta_{1}, \zeta_{2}, \ldots, \zeta_{\rho-1}$ bezeichnet. Mit $\tilde{\boldsymbol{x}}=\left[\begin{array}{lll}\boldsymbol{x}^{\top} & \boldsymbol{u}^{\top} \boldsymbol{\zeta}_{1}^{\top} \ldots \boldsymbol{\zeta}_{\rho-1}^{\top}\end{array}\right]^{\top} \in \mathbb{R}^{n+\rho n_{u}}$ ergibt sich daraus die um die Reglerzustände erweiterte Strecke

$\dot{\tilde{\boldsymbol{x}}}=\underbrace{\left[\begin{array}{ccccc}\boldsymbol{A} & \boldsymbol{B} & \mathbf{0} & \cdots & \mathbf{0} \\ \mathbf{0} & \mathbf{0} & \boldsymbol{I}_{n_{u}} & & \vdots \\ \vdots & & \ddots & \ddots & \\ \mathbf{0} & \cdots & \cdots & \mathbf{0} & \boldsymbol{I}_{n_{u}} \\ \mathbf{0} & \cdots & \cdots & \cdots & \mathbf{0}\end{array}\right]}_{\widetilde{\boldsymbol{A}}} \tilde{\boldsymbol{x}+} \underbrace{\left[\begin{array}{c}\mathbf{0} \\ \vdots \\ \vdots \\ \mathbf{0} \\ \boldsymbol{I}_{n_{u}}\end{array}\right]}_{\widetilde{\boldsymbol{B}}} \zeta_{\rho}$,

$y=\underbrace{\left[\begin{array}{llll}C & 0 & \cdots & 0\end{array}\right]}_{\widetilde{C}} \tilde{x}$.

Dabei gilt

Lemma 1. Die um $\rho$ vorgeschaltete Integratorblöcke erweiterte Strecke $(\widetilde{\boldsymbol{A}}, \widetilde{\boldsymbol{B}}, \widetilde{\boldsymbol{C}})$ ist genau dann statisch stabil entkoppelbar, wenn $(\boldsymbol{A}, \boldsymbol{B}, \boldsymbol{C})$ statisch stabil entkoppelbar ist.

Beweis 1. Aufgrund der vorgeschalteten Integratorkette sind die Differenzenordnungen der einzelnen Übertragungskanäle jeweils um $\rho$ erhöht. Dies lässt sich auch anhand der Definitionsgleichung der Differenzenordnungen (11) für das erweiterte System zeigen. Es gilt $\tilde{\boldsymbol{c}}_{i}^{\top} \widetilde{\boldsymbol{A}}^{k} \widetilde{\boldsymbol{B}}=\mathbf{0}^{\top}$ für $k=0, \ldots, \rho-1 . \mathrm{Da}$

$\widetilde{\boldsymbol{A}}^{\rho+k}=\left[\begin{array}{cccc}\boldsymbol{A}^{\rho+k} & \boldsymbol{A}^{\rho+k-1} \boldsymbol{B} & \cdots & \boldsymbol{A}^{k} \boldsymbol{B} \\ \mathbf{0} & \cdots & \cdots & \mathbf{0} \\ \vdots & \ddots & & \vdots \\ \vdots & & \ddots & \vdots \\ \mathbf{0} & \cdots & \cdots & \mathbf{0}\end{array}\right]$

gilt, erhält man

$\tilde{\boldsymbol{c}}_{i}^{\top} \widetilde{\boldsymbol{A}}^{k} \widetilde{\boldsymbol{B}}=\boldsymbol{c}_{i}^{\top} \boldsymbol{A}^{k-\rho} \boldsymbol{B} \forall k=\rho, \ldots, \rho+\delta_{i}-1$.

Demnach ist $\tilde{\boldsymbol{c}}_{i}^{\top} \widetilde{\boldsymbol{A}}^{\rho+\delta_{i}-1} \widetilde{\boldsymbol{B}}=\boldsymbol{c}_{i}^{\top} \boldsymbol{A}^{\delta_{i}-1} \boldsymbol{B} \neq \mathbf{0}^{\top}$ und es gilt $\widetilde{D}^{*}=D^{*}$. Folglich ändert sich die Entkoppelbarkeitsmatrix im erweiterten System gegenüber dem ursprünglichen System nicht (s. (12)). Damit ist die statische Entkoppelbarkeit des erweiterten Systems gezeigt. 
Um auch die stabile Entkoppelbarkeit nachzuweisen, wird die Rosenbrock'sche Systemmatrix des erweiterten Systems zu

$$
\begin{aligned}
\widetilde{\boldsymbol{P}}(s)= & {\left[\begin{array}{ccc}
s \boldsymbol{I}_{n+\rho \eta_{u}}-\widetilde{\boldsymbol{A}} & -\widetilde{\boldsymbol{B}} \\
\widetilde{\boldsymbol{C}} & \mathbf{0}
\end{array}\right] } \\
= & {\left[\begin{array}{ccccc}
s \boldsymbol{I}_{n}-\boldsymbol{A} & -\boldsymbol{B} & \mathbf{0} & \ldots & \mathbf{0} \\
\mathbf{0} & s \boldsymbol{I}_{n_{u}} & -\boldsymbol{I}_{n_{u}} & & \vdots \\
\vdots & & \ddots & \ddots & \\
\mathbf{0} & \ldots & \cdots & s \boldsymbol{I}_{n_{u}} & -\boldsymbol{I}_{n_{u}} \\
\boldsymbol{C} & \mathbf{0} & \cdots & \cdots & \mathbf{0}
\end{array}\right] . }
\end{aligned}
$$

berechnet. Durch sukzessives Entwickeln von $\operatorname{det} \widetilde{\boldsymbol{P}}(s)$ nach Spalten, die jeweils nur ein Nichtnullelement enthalten, folgt

$\operatorname{det} \widetilde{\boldsymbol{P}}(s)=\left|\begin{array}{cc}s \boldsymbol{I}_{n}-\boldsymbol{A} & -\boldsymbol{B} \\ \boldsymbol{C} & \mathbf{0}\end{array}\right|=\operatorname{det} \boldsymbol{P}(s)$,

was impliziert, dass die Invarianten Nullstellen von $(\widetilde{\boldsymbol{A}}, \widetilde{\boldsymbol{B}}, \widetilde{\boldsymbol{C}})$ mit denen von $(\boldsymbol{A}, \boldsymbol{B}, \boldsymbol{C})$ übereinstimmen [29]. $\mathrm{Da}$ die Minimalphasigkeit von $(\boldsymbol{A}, \boldsymbol{B}, \boldsymbol{C})$ vorausgesetzt wird, ist also auch $(\widetilde{\boldsymbol{A}}, \widetilde{\boldsymbol{B}}, \widetilde{\boldsymbol{C}})$ statisch stabil entkoppelbar.

Der Entwurf des Reglers erfolgt analog zu [10]. Bildet man die zeitlichen Ableitungen der einzelnen Ausgänge, so ergibt sich aufgrund der in Beweis 1 bestimmten Differenzenordnungen

$$
\begin{aligned}
y_{i} & =\tilde{\boldsymbol{c}}_{i}^{\top} \tilde{\boldsymbol{x}}=\boldsymbol{c}_{i}^{\top} \boldsymbol{x}, \\
\dot{y}_{i} & =\tilde{\boldsymbol{c}}_{i}^{\top} \widetilde{\boldsymbol{A}} \tilde{\boldsymbol{x}}, \\
\vdots & \\
y_{i}^{\left(\rho+\delta_{i}-1\right)} & =\tilde{\boldsymbol{c}}_{i}^{\top} \widetilde{\boldsymbol{A}}^{\rho+\delta_{i}-1} \tilde{\boldsymbol{x}}, \\
y_{i}^{\left(\rho+\delta_{i}\right)} & =\tilde{\boldsymbol{c}}_{i}^{\top} \widetilde{\boldsymbol{A}}^{\rho+\delta_{i}} \tilde{\boldsymbol{x}}+\tilde{\boldsymbol{c}}_{i}^{\top} \widetilde{\boldsymbol{A}}^{\rho+\delta_{i}-1} \widetilde{\boldsymbol{B}} \boldsymbol{\zeta}_{\rho} \\
& =\tilde{\boldsymbol{c}}_{i}^{\top} \widetilde{\boldsymbol{A}}^{\rho+\delta_{i}} \tilde{\boldsymbol{x}}+\boldsymbol{c}_{i}^{\top} \boldsymbol{A}^{\delta_{i}-1} \boldsymbol{B} \boldsymbol{\zeta}_{\rho} .
\end{aligned}
$$

Die Transformation von (14) in den Zeitbereich führt auf die Forderung

$y_{i}^{\left(\rho+\delta_{i}\right)}+q_{i \rho+\delta_{i}-1} y_{i}^{\left(\rho+\delta_{i}-1\right)}+\ldots+q_{i 0} y_{i}=p_{i 0} w_{i}$.

Setzt man in diese Beziehung die zeitlichen Ableitungen (20) ein und fasst die Bedingungen für alle Übertragungskanäle zusammen, so ergibt sich

$\tilde{\boldsymbol{M}} \tilde{\boldsymbol{x}}+D^{*} \zeta_{\rho}=\tilde{N} w$

mit

$\tilde{\boldsymbol{M}}=\left[\begin{array}{c}\tilde{\boldsymbol{c}}_{1}^{\top} \widetilde{\boldsymbol{A}}^{\rho+\delta_{1}}+\sum_{k=0}^{\rho+\delta_{1}-1} q_{1 k} \tilde{\boldsymbol{c}}_{1}^{\top} \widetilde{\boldsymbol{A}}^{k} \\ \vdots \\ \tilde{\boldsymbol{c}}_{n_{y}}^{\top} \widetilde{\boldsymbol{A}}^{\rho+\delta_{n y}}+\sum_{k=0}^{\rho+\delta_{n_{y}}-1} q_{n_{y} k} \tilde{\boldsymbol{c}}_{n_{y}}^{\top} \widetilde{\boldsymbol{A}}^{k}\end{array}\right]$,

$\widetilde{N}=\operatorname{diag}\left(p_{10}, \ldots, p_{n_{y} 0}\right)$.
Da die statische Entkoppelbarkeit von $(\boldsymbol{A}, \boldsymbol{B}, \boldsymbol{C})$ vorausgesetzt wurde, lässt sich das Regelgesetz für das erweiterte System nun unmittelbar als

$\zeta_{\rho}=-\underbrace{D^{*-1} \tilde{M}}_{\widetilde{K}} \tilde{x}+\underbrace{D^{*-1} \tilde{N}}_{\widetilde{F}} w$

angeben.

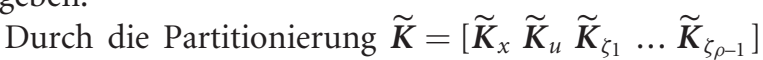
lässt sich mit $\boldsymbol{\xi}=\left[\begin{array}{lll}\boldsymbol{u}^{\top} \boldsymbol{\zeta}_{1}^{\top} \ldots \boldsymbol{\zeta}_{\rho-1}^{\top}\end{array}\right]^{\top} \in \mathbb{R}^{n_{c}}, n_{c}=\rho n_{u}$ eine Realisierung des dynamischen Zustandsreglers

$\dot{\xi}=\boldsymbol{A}_{\xi} \boldsymbol{\xi}+\boldsymbol{B}_{\xi, x} \boldsymbol{x}+\boldsymbol{B}_{\xi, w} \boldsymbol{w}$,

$\boldsymbol{u}=C_{\xi} \boldsymbol{\xi}+\boldsymbol{D}_{\xi, x} \boldsymbol{x}+\boldsymbol{D}_{\xi, w} \boldsymbol{w}$

mit

$\boldsymbol{A}_{\xi}=\left[\begin{array}{ccccc}\mathbf{0} & \boldsymbol{I}_{n_{u}} & \mathbf{0} & \cdots & \mathbf{0} \\ \vdots & & \ddots & & \vdots \\ \vdots & & & \ddots & \vdots \\ \mathbf{0} & \cdots & \cdots & \mathbf{0} & \widetilde{I}_{n_{u}} \\ -\widetilde{\boldsymbol{K}}_{u} & -\widetilde{\boldsymbol{K}}_{\zeta_{1}} & -\widetilde{\boldsymbol{K}}_{\zeta_{2}} & \cdots & -\widetilde{\boldsymbol{K}}_{\zeta_{\rho-1}}\end{array}\right]$,

$\boldsymbol{B}_{\xi, x}=\left[\begin{array}{llll}\mathbf{0} & \cdots & \mathbf{0} & -\widetilde{\boldsymbol{K}}_{x}^{\top}\end{array}\right]^{\top}$,

$\boldsymbol{B}_{\xi, w}=\left[\begin{array}{llll}\mathbf{0} & \cdots & \mathbf{0} & \widetilde{\boldsymbol{F}}^{\top}\end{array}\right]^{\top}$,

$C_{\xi}=\left[\begin{array}{llll}I_{n_{u}} & \mathbf{0} & \cdots & \mathbf{0}\end{array}\right]$,

$\boldsymbol{D}_{\xi, x}=\mathbf{0}, \boldsymbol{D}_{\xi, w}=\mathbf{0}$

angeben.

\section{Dualitätsbasierter Entwurf dynamischer FIOs}

In ähnlicher Art und Weise, wie in [18] statische FIOs entworfen werden, lassen sich dynamische FIOs durch Ausnutzung der Dualität zu Entkopplungsregelungen entwerfen (s. Bild 1). Analog zur Differenzenordnung einzelner Ausgänge werden dabei die Fehlerdetektionsindizes $\mu_{i}$ definiert als $\mu_{i}=\min _{k}\left\{k \geq 1: C A^{k-1} \boldsymbol{e}_{a_{i}} \neq \mathbf{0}\right\}$ [18]. Dabei beschreibt $\boldsymbol{e}_{a_{i}}$ die $i$-te Spalte der Fehlereingangsmatrix $\boldsymbol{E}_{a}$. Ebenfalls basierend auf der Dualität wird die Fehlerdetektionsmatrix

$D_{L}^{*}=\left[\boldsymbol{d}_{L, 1}^{*} \cdots \boldsymbol{d}_{L, n_{y}}^{*}\right]$,

$\boldsymbol{d}_{L, i}^{*}=C A^{\mu_{i}-1} \boldsymbol{e}_{a_{i}}$

eingeführt. Während die Ergebnisse in [18] auf Systeme mit $\mu_{i}=1 \forall i=1, \ldots, n_{y}$ beschränkt sind, gelten die hier erzielten Resultate für beliebige Fehlerdetektionsindizes.

Um den Entwurf von FIOs auch im dynamisch erweiterten Fall auf Entkopplungsregelungen zurückzuführen, wird zunächst die Dynamik einer mittels eines dynamischen Reglers (25) geregelten Strecke (1) betrachtet, bei 
$\operatorname{der} \boldsymbol{f}=\mathbf{0}$ und $\boldsymbol{d}=\mathbf{0}$ gelten. Sie lautet

$$
\begin{gathered}
{\left[\begin{array}{c}
\dot{\boldsymbol{x}} \\
\dot{\xi}
\end{array}\right]=\left(\left[\begin{array}{cc}
\boldsymbol{A} & \mathbf{0} \\
\mathbf{0} & \mathbf{0}
\end{array}\right]+\left[\begin{array}{cc}
\boldsymbol{B} & \mathbf{0} \\
\mathbf{0} & \boldsymbol{I}_{n_{c}}
\end{array}\right]\left[\begin{array}{ll}
\boldsymbol{D}_{\xi, x} & C_{\xi} \\
\boldsymbol{B}_{\xi, x} & \boldsymbol{A}_{\xi}
\end{array}\right]\right)\left[\begin{array}{l}
\boldsymbol{x} \\
\boldsymbol{\xi}
\end{array}\right]} \\
+\left[\begin{array}{cc}
\boldsymbol{B} & \mathbf{0} \\
\mathbf{0} & \boldsymbol{I}_{n_{c}}
\end{array}\right]\left[\begin{array}{l}
\boldsymbol{D}_{\xi, w} \\
\boldsymbol{B}_{\xi, w}
\end{array}\right] w, \\
\boldsymbol{y}=\left[\begin{array}{ll}
\boldsymbol{C} & \mathbf{0}
\end{array}\right]\left[\begin{array}{l}
\boldsymbol{x} \\
\boldsymbol{\xi}
\end{array}\right] .
\end{gathered}
$$

Demgegenüber ergibt sich mit $\boldsymbol{e}=\boldsymbol{x}-\hat{\boldsymbol{x}}$ die Dynamik des dynamischen Beobachters und der Residuengenerierung (5) zu

$$
\begin{gathered}
{\left[\begin{array}{c}
\dot{\boldsymbol{e}} \\
\dot{z}
\end{array}\right]=\left(\left[\begin{array}{cc}
A & 0 \\
0 & 0
\end{array}\right]-\left[\begin{array}{cc}
L_{p} & \boldsymbol{L}_{i} \\
-\boldsymbol{\Gamma} & -\boldsymbol{\Phi}
\end{array}\right]\left[\begin{array}{cc}
\boldsymbol{C} & 0 \\
\mathbf{0} & \boldsymbol{I}_{\nu}
\end{array}\right]\right)\left[\begin{array}{l}
\boldsymbol{e} \\
z
\end{array}\right]} \\
+\left[\begin{array}{c}
\boldsymbol{E}_{a} \\
\mathbf{0}
\end{array}\right] f \\
\boldsymbol{r}=\left[\begin{array}{ll}
\boldsymbol{V}_{1} & \boldsymbol{V}_{2}
\end{array}\right]\left[\begin{array}{ll}
\boldsymbol{C} & \mathbf{0} \\
\mathbf{0} & \boldsymbol{I}_{\nu}
\end{array}\right]\left[\begin{array}{l}
\boldsymbol{e} \\
z
\end{array}\right],
\end{gathered}
$$

wobei hier nur der Einfluss der Fehler berücksichtigt wird, d. h. $\boldsymbol{d}=\mathbf{0}$. Das duale System ist damit durch

$$
\begin{aligned}
& {\left[\begin{array}{c}
\dot{\overline{\boldsymbol{e}}} \\
\dot{\bar{z}}
\end{array}\right]=}\left(\left[\begin{array}{cc}
\boldsymbol{A}^{\top} & \mathbf{0} \\
\mathbf{0} & \mathbf{0}
\end{array}\right]+\left[\begin{array}{cc}
\boldsymbol{C}^{\top} & \mathbf{0} \\
\mathbf{0} & \boldsymbol{I}_{v}
\end{array}\right]\left[\begin{array}{cc}
-\boldsymbol{L}_{p}^{\top} & \boldsymbol{\Gamma}^{\top} \\
-\boldsymbol{L}_{i}^{\top} & \boldsymbol{\Phi}^{\top}
\end{array}\right]\right)\left[\begin{array}{c}
\overline{\boldsymbol{e}} \\
\bar{z}
\end{array}\right] \\
&+\left[\begin{array}{cc}
\boldsymbol{C}^{\top} & \mathbf{0} \\
\mathbf{0} & \boldsymbol{I}_{v}
\end{array}\right]\left[\begin{array}{l}
\boldsymbol{V}_{1}^{\top} \\
\boldsymbol{V}_{2}^{\top}
\end{array}\right] \boldsymbol{f}, \\
& \boldsymbol{r}=\left[\begin{array}{ll}
\boldsymbol{E}_{a}^{\top} & \mathbf{0}
\end{array}\right]\left[\begin{array}{c}
\overline{\boldsymbol{e}} \\
\overline{\boldsymbol{z}}
\end{array}\right]
\end{aligned}
$$

gegeben. Der Vergleich von (28) und (30) verdeutlicht, dass ein dynamischer Fehlerisolationsbeobachter mit der Dynamik (14) in den Diagonalelementen durch den Entwurf eines dynamischen Entkopplungsreglers wie in Abschnitt 4 für das System $\left(\boldsymbol{A}^{\top}, \boldsymbol{C}^{\top}, \boldsymbol{E}_{a}^{\top}\right)$ gefunden werden kann. Die Substitutionen $\boldsymbol{\Phi}=\boldsymbol{A}_{\xi}^{\top}, \boldsymbol{\Gamma}=\boldsymbol{C}_{\xi}^{\top}$, $\boldsymbol{L}_{p}=-\boldsymbol{D}_{\xi, x}^{\top}, \boldsymbol{L}_{i}=-\boldsymbol{B}_{\xi, x}^{\top}, \boldsymbol{V}_{1}=\boldsymbol{D}_{\xi, w}^{\top}$ und $\boldsymbol{V}_{2}=\boldsymbol{B}_{\xi, w}^{\top}$ liefern schließlich die Parametrierung des dynamischen FIO.

\subsection{Unterdrückung hochfrequenter Störungen}

Im Folgenden wird die Übertragungsmatrix von Störungen auf die Residuen betrachtet, also $\boldsymbol{G}_{\boldsymbol{r d}}(s)=$ $C_{r d}\left(s \boldsymbol{I}_{n+v}-\boldsymbol{A}_{r d}\right)^{-1} \boldsymbol{B}_{r d}+\boldsymbol{D}_{r d}$, mit

$$
\begin{array}{rlr}
A_{r d} & =\left[\begin{array}{cc}
A-L_{p} C & -L_{i} \\
\Gamma C & \Phi
\end{array}\right], & B_{r d}=\left[\begin{array}{c}
B_{d}-L_{p} D_{d} \\
\Gamma D_{d}
\end{array}\right], \\
C_{r d}=\left[\begin{array}{ll}
V_{1} C & V_{2}
\end{array}\right], & D_{r d}=V_{1} D_{d} .
\end{array}
$$

Nach (8) ist $\boldsymbol{A}_{r f}=\boldsymbol{A}_{\boldsymbol{r} \boldsymbol{d}}$, demnach verfügen die Elemente von $\boldsymbol{G}_{\boldsymbol{r} \boldsymbol{d}}(s)$ und $\boldsymbol{G}_{\boldsymbol{r} f}(s)$ über denselben Nenner. Die Grundidee ist es nun, für die Diagonalpfade

$$
r_{i}(s)=\frac{p_{i 0}}{s^{\mu_{i}+\rho}+\cdots+q_{i 1} s+q_{i 0}} f_{i}(s)
$$

vorzugeben, wodurch der relative Grad der Diagonalelemente gegenüber statischen FIOs um $\rho$ erhöht wird. Dies führt folglich zu zusätzlichen Polen in $\boldsymbol{G}_{\boldsymbol{r d}}(s)$, was wiederum einen Abfall der Verstärkung bei hohen Frequenzen und damit eine bessere Störunterdrückung bedingt.

Für stabil fehlerisolierbare Systeme, d.h. wenn Annahme 1 erfüllt ist, lässt sich ein vergleichbares Übertragungsverhalten $\boldsymbol{G}_{\boldsymbol{r} d}(s)$ erreichen, indem ein statischer FIO implementiert wird und die so generierten Residuen anschließend wie z. B. in [25] gefiltert werden. Es können also mittels eines Beobachters der Form (2) Residuen

$$
\overline{\boldsymbol{r}}(s)=\operatorname{diag}\left(\bar{g}_{11}(s), \ldots, \bar{g}_{n_{f} n_{f}}(s)\right)
$$

generiert werden, die anschließend durch eine Bank von stabilen Postfiltern in

$$
\begin{aligned}
r(s) & =\operatorname{diag}\left(\bar{R}_{1}(s), \ldots, \bar{R}_{n_{f}}(s)\right) \overline{\boldsymbol{r}}(s) \\
& =\operatorname{diag}\left(g_{11}(s), \ldots, g_{n_{f} n_{f}}(s)\right)
\end{aligned}
$$

überführt werden. Die hier vorgestellte Methode hat den Vorteil, dass konjugiert komplexe Polpaare auch für Übertragungspfade mit ungerader Differenzenordnung vorgegeben werden können. Eine nachträgliche Filterung der Residuen würde in solchen Fällen eine erhöhte Filterordnung und damit einen höheren Implementierungsaufwand bedingen. Darüber hinaus entfällt bei dem hier vorgestellten Ansatz der separate Entwurf von statischem FIO und zusätzlichem Filter, sodass er sich im allgemeinen Framework dynamischer Beobachter [24] darstellen lässt. Weiterhin kann ein statischer FIO stark verrauschte Residuen generieren, die erst nachträglich geglättet werden. Der integrierte Ansatz dagegen vermeidet solche Signale mit möglicherweise hohen Amplituden und die damit u. U. verbundenen Implementierungsprobleme.

Anmerkung 2. Anzumerken ist überdies, dass bei statischen FIOs der Form (2) durch stabile Postfilter wie in (34) verschiedene Verarbeitungsschritte der Residuen vorgenommen werden können. Ein Beispiel ist die näherungsweise Differentiation der Residuen zur Trendanalyse. Diese lassen sich auch direkt in den Entwurf des dynamischen FIO integrieren. Dafür ist jedoch in Verallgemeinerung von (14) auch die Ordnung der Zähler der einzelnen Übertragungspfade zu erhöhen, worauf im Rahmen dieses Beitrages nicht näher eingegangen wird.

Anmerkung 3. Zu erwähnen ist weiterhin, dass mit dem in Anmerkung 2 beschriebenen Frequenzbereichsentwurf auch der Entwurf von dynamischen FIOs für statisch nicht fehlerisolierbare Systeme möglich ist. Ähnlich wie in [19] ist dazu die Struktur des orthogonalen Komplements von $\boldsymbol{D}_{L}^{*}$ zu untersuchen. Die ausführliche Untersuchung dieser Thematik ist jedoch nicht Gegenstand dieses Artikels, sondern wird in [31] erörtert. 


\subsection{Optimierung der Detektionsgeschwindigkeit bei vorgegebener Störunterdrückung}

Wie in Abschnitt 5.1 erörtert, lässt sich durch die Erhöhung der Nennerordnung in den einzelnen Übertragungspfaden eine verbesserte Unterdrückung hochfrequenter Störungen erreichen. Ein alternatives Entwurfsziel ist die Steigerung der Detektionsgeschwindigkeit unter Einhaltung vorgegebener Schranken für die Verstärkung hochfrequenter Störungen.

$\mathrm{Zu}$ diesem Zweck wird das verallgemeinerte KalmanYakubovich-Popov (KYP) Lemma herangezogen. Es ermöglicht die Formulierung von LMI-Bedingungen zur Abschätzung der $H_{\infty}$-Norm einer Übertragungsmatrix in einem beschränkten Frequenzbereich. Im Bereich der beobachterbasierten Fehlerdetektion wird dieser Ansatz in einer Vielzahl aktueller Publikationen verwendet (s. z. B. $[6 ; 16 ; 32-34]$ ).

In diesem Beitrag wird das verallgemeinerte KYP Lemma für die Fehlerisolation angewendet und wird in Anlehnung an [14] in folgender Form benötigt.

Lemma 2. Gegeben sei ein stabiles System der Form

$\dot{x}=A x+B u$,

$y=C x+D u$

mit $\boldsymbol{x} \in \mathbb{R}^{n}, \boldsymbol{u} \in \mathbb{R}^{n_{u}}, \boldsymbol{y} \in \mathbb{R}^{n_{y}}$, Matrizen entsprechender Dimensionen und der Übertragungsmatrix $G(s)=C(s I-$ $\boldsymbol{A})^{-1} \boldsymbol{B}+\boldsymbol{D}$. Weiterhin sei $\Omega=\left[\omega_{l}, \infty\right)$ und $\beta>0$. Dann sind die folgenden Aussagen äquivalent:

1. $\|\boldsymbol{G}(s)\|_{\infty}^{\Omega}<\beta$.

2. $\left[\begin{array}{c}\boldsymbol{G}(j \omega) \\ \boldsymbol{I}\end{array}\right]^{H} \boldsymbol{\Pi}\left[\begin{array}{c}\boldsymbol{G}(j \omega) \\ \boldsymbol{I}\end{array}\right] \prec \mathbf{0} \quad \forall \omega \in \Omega$, mit $\boldsymbol{\Pi}=\left[\begin{array}{cc}\boldsymbol{I} & \mathbf{0} \\ \mathbf{0} & -\beta^{2} \boldsymbol{I}\end{array}\right]$.

3. Es existieren hermitesche Matrizen $\boldsymbol{X} \succ \mathbf{0}$ und $\boldsymbol{Y}$, sodass

$$
\begin{aligned}
& {\left[\begin{array}{cc}
\boldsymbol{A} & \boldsymbol{B} \\
\boldsymbol{I} & \mathbf{0}
\end{array}\right]^{H} \boldsymbol{\Xi}\left[\begin{array}{cc}
\boldsymbol{A} & \boldsymbol{B} \\
\boldsymbol{I} & \mathbf{0}
\end{array}\right]+\left[\begin{array}{cc}
\boldsymbol{C} & \boldsymbol{D} \\
\mathbf{0} & \boldsymbol{I}
\end{array}\right]^{H} \boldsymbol{\Pi}\left[\begin{array}{cc}
\boldsymbol{C} & \boldsymbol{D} \\
\mathbf{0} & \boldsymbol{I}
\end{array}\right] \prec \mathbf{0},} \\
& \text { mit } \boldsymbol{\Xi}=\left[\begin{array}{cc}
\boldsymbol{X} & \boldsymbol{Y} \\
\boldsymbol{Y} & -\omega_{l}^{2} \boldsymbol{X}
\end{array}\right], \quad \boldsymbol{\Pi}=\left[\begin{array}{cc}
\boldsymbol{I} & \mathbf{0} \\
\mathbf{0} & -\beta^{2} \boldsymbol{I}
\end{array}\right] \text { gilt. }
\end{aligned}
$$

Auf dieser Grundlage sei nun angenommen, dass entsprechend Abschnitt 5.1 ein dynamischer FIO entworfen wurde, der die Pole $\lambda_{i j}$ mit $i=1, \ldots, n_{f}, j=1, \ldots, \mu_{i}+\rho$ und Zählerkoeffizienten $p_{i 0}$ erzeugt. Wird Lemma 2 auf das durch (31) definierte System angewendet, so lässt sich für einen durch $\omega_{l}$ festgelegten Frequenzbereich eine obere Schranke $\beta_{0}$ ermitteln, sodass $\left\|\boldsymbol{G}_{\boldsymbol{r} \boldsymbol{d}}(s)\right\|_{\infty}^{\left[\omega_{l}, \infty\right)}<\beta_{0}$ erfüllt ist. Offensichtlich kann die Detektionsgeschwindigkeit erhöht werden, indem die vorgegebenen Pole weiter links in der s-Halbebene platziert werden. Sind jedoch hochfrequente Störungen vorhanden, so bedingt dies für $\boldsymbol{D}_{d} \neq \mathbf{0}$ i. A. eine größere Verstärkung der Störungen. Ist für die maximale Verstärkung der Störungen eine obere Schranke $\beta_{\text {lim }}>\beta_{0}$ vorgegeben, so lässt sich ein

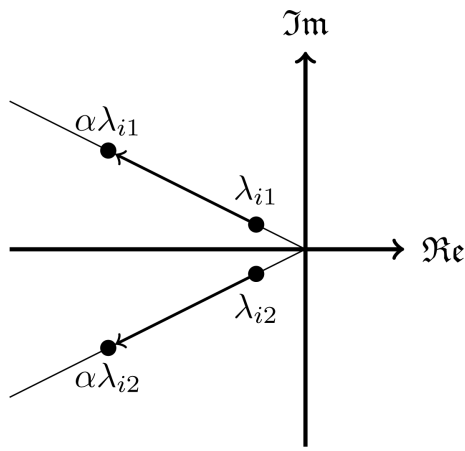

Bild 3 Skalierte Pole zur Erhöhung der Detektionsgeschwindigkeit.

Kompromiss zwischen den konkurrierenden Anforderungen erzielen. Dazu werden, wie in Bild 3 verdeutlicht, die Pole mit einem Faktor $\alpha>0$ skaliert, wobei die Störverstärkung $\left\|\boldsymbol{G}_{\boldsymbol{r d}}(s)\right\|_{\infty}^{\left[\omega_{l}, \infty\right)}$ den Wert $\beta_{\text {lim }}$ nicht überschreiten darf. Dies kann als Optimierungsproblem

maximiere $\alpha$, sodass

$\boldsymbol{G}_{r f}(s)=\operatorname{diag}\left(g_{11}(s), \cdots, g_{n_{f} n_{f}}(s)\right)$,

$g_{i i}(s)=\frac{(-\alpha)^{\mu_{i}+\rho} p_{i 0}}{\left(s-\alpha \lambda_{i 1}\right) \cdot \ldots \cdot\left(s-\alpha \lambda_{i \mu_{i}+\rho}\right)}$,

$\left\|\boldsymbol{G}_{\boldsymbol{r d}}(s)\right\|_{\infty}^{\left[\omega_{l} \infty\right)}<\beta_{\lim }$

formuliert werden. Dabei wird vorausgesetzt, dass $\left\|\boldsymbol{G}_{\boldsymbol{r} \boldsymbol{d}}(s)\right\|_{\infty}^{[\omega, \infty)}$ monoton steigend in $\alpha$ ist. Um eine gleichbleibende statische Verstärkung der Fehler zu gewährleisten, werden die Zählerkoeffizienten in (35b) ebenfalls skaliert. Sollen dagegen Fehler beliebiger Frequenzen berücksichtigt werden, bietet sich alternativ die Formulierung eines $\mathcal{H}_{i} / \mathcal{H}_{\infty}$-Problems [9] an. Das Optimierungsproblem (35) wird gelöst, indem zunächst ein $\alpha^{*}$ ermittelt wird, welches $\beta<\beta_{\text {lim }}$ verletzt. Daraufhin wird der größte zulässige Wert $\alpha_{\max }$ im Intervall $\left[1, \alpha^{*}\right)$ ermittelt. Dies erfolgt in

\section{Algorithmus 1.}

1. Wähle $\rho$ und entwerfe einen dynamischen FIO gemäß Abschnitt 5.1 mit vorgegebenen Polen $\lambda_{i j}$ und Koeffizienten $p_{i 0}$, sodass $\beta_{0}<\beta_{\text {lim. }}$. Setze $\alpha=1$.

2. Setze $\alpha^{*}=2 \cdot \alpha$ und entwerfe einen dynamischen FIO mit Polen $\lambda_{i j}^{*}=\alpha^{*} \cdot \lambda_{i j}$ entsprechend (35b). Wenn dieser FIO $\beta<\beta_{\text {lim }}$ erfüllt, setze $\alpha:=\alpha^{*}$ und gehe zu Schritt 2. Andernfalls gehe zu Schritt 3.

3. Ermittle größten zulässigen Wert $\alpha_{\max }$ in $\left[\alpha=1, \alpha^{*}\right)$ mittels Bisektion.

Da $\rho$ ein Entwurfsparameter ist, kann er frei gewählt werden. Für $\rho=0$ wird ein statischer FIO der Form (2) entworfen. Größere Werte für $\rho$ führen zu einer verbesserten Unterdrückung hochfrequenter Störungen, erhöhen jedoch die Ordnung des Fehlerisolationssystems. 

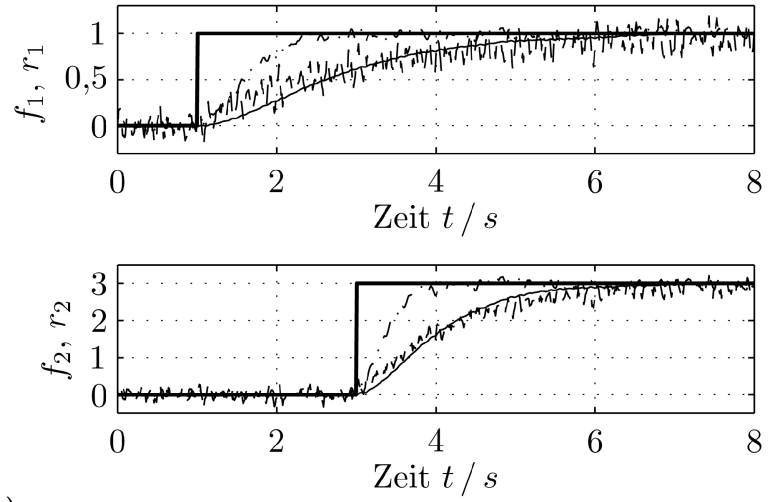

a)

Bild 4 Simulationsergebnisse; (a) Zeitverlauf der Fehler (-) und Residuen (-- statischer FIO, - dynamischer FIO, - - - optimierter dynamischer FIO), (b) Amplitudengang von $\boldsymbol{G}_{r d}(s)$ (-- statischer FIO, - dynamischer FIO, - - - optimierter dynamischer FIO).

Schritt 3 setzt ein geeignetes Abbruchkriterium voraus. Hier bietet sich eine Schranke für die Schrittweite der Bisektion an. Da angenommen wird, dass $\left\|\boldsymbol{G}_{\boldsymbol{r} \boldsymbol{d}}(s)\right\|_{\infty}^{\left[\omega_{l}, \infty\right)}$ monoton in $\alpha$ ansteigt, ist die Konvergenz von Algorithmus 1 sichergestellt.

\section{Beispiel}

Zur Verdeutlichung der Ergebnisse wird ein dynamischer FIO für ein Strahltriebwerk entworfen. Das Modell ist [15] entnommen, es handelt sich um ein lineares System mit $n=n_{y}=n_{f}=2$, definiert durch

$A=\left[\begin{array}{rr}-3,370 & 1,636 \\ -0,325 & -1,896\end{array}\right], \quad C=\left[\begin{array}{cc}1 & 0 \\ 0,731 & 0,786\end{array}\right]$.

Zustände des Systems sind die Drehzahlen von Niederund Hochdruckturbine, gemessen werden die Drehzahl der Niederdruckturbine sowie der statische Druck am Brennkammerausgang. Die Fehler- und Störeingangsmatrizen sind

$\boldsymbol{B}_{d}=\left[\begin{array}{ll}-0,216 & 0,062 \\ -0,833 & 0,144\end{array}\right], \boldsymbol{D}_{d}=\left[\begin{array}{rr}-0,573 & -0,019 \\ 0,596 & 0,087\end{array}\right]$,

$\boldsymbol{E}_{a}=\boldsymbol{B}=\left[\begin{array}{rr}0,586 & -1,419 \\ 0,410 & 1,118\end{array}\right]$

und als Störungen werden

$\boldsymbol{d}(t)=\left[\begin{array}{c}0,2 \sin (2 \pi 8 t)+0,15 \sin (2 \pi 10 t) \\ 0,1 \sin (2 \pi 7 t)+0,2 \sin (2 \pi 9 t)\end{array}\right]+0,1 \boldsymbol{\mu}(t)$,

angenommen. Dabei ist $\boldsymbol{\mu}(t)$ weißes mittelwertfreies Gauß'sches Rauschen mit einer Standardabweichung von 1. Mögliche Fehler können in der zugeführten Kraftstoffmenge $\left(f_{1}\right)$ und in der Stellung der Leitschaufel $\left(f_{2}\right)$ auftreten. Zu Vergleichszwecken wird ein statischer FIO mit dem Pol $\sigma_{\text {stat }, 1}=\{-0,5\}$ für den ersten Kanal und $\sigma_{\text {stat }, 2}=\{0,8\}$ für den zweiten entworfen, die statische Verstärkung ist jeweils 1. Entsprechend Abschnitt 5.1 wird ein dynamischer Beobachter entworfen, welcher die Pole $\sigma_{\text {dyn, } 1}=\{-1 \pm 0,2 J\}$ beziehungsweise $\sigma_{\text {dyn }, 2}=\{-1,7 ;-1,9\}$ erzeugt. Bild 4a zeigt die generierten Residuen beider Beobachter bei sprungförmigen Fehlern $f_{1}$ und $f_{2}$, die zu den Zeitpunkten $t_{1}=1 \mathrm{~s}$ und $t_{2}=3 \mathrm{~s}$ auftreten. Bei vergleichbarer Detektionsgeschwindigkeit erzeugt der dynamische Beobachter wesentlich weniger gestörte Residuen, was in der verbesserten Störunterdrückung (s. Bild $4 \mathrm{~b}$ ) begründet ist. Da lediglich hochfrequente Störungen auftreten, wird $\omega_{l}=31,42 \mathrm{rad} / \mathrm{s}$ zur Berechnung von $\left\|\boldsymbol{G}_{\boldsymbol{r} \boldsymbol{d}}(s)\right\|_{\infty}^{\Omega}$ verwendet. Mittels YALMIP [20] und des LMI-Solvers SDPT3 [30] ergeben sich $\beta_{\text {stat }}=0,806$ und $\beta_{0}=\beta_{\text {dyn }}=0,089$. Mithilfe von Algorithmus 1 und einer vorgegebenen Schranke $\beta_{\text {lim }}=0,7$ ergibt sich ein zulässiger Skalierungsfaktor $\alpha_{\max }=2,805$. Die Pole des optimierten dynamischen Beobachters sind dann $\sigma_{\text {dyn,opt }, 1}=\{-2,805 \pm 0,561 \mathrm{~J}\}$ und $\sigma_{\text {dyn,opt }, 2}=\{-4,768 ;-5,329\}$ und es ergeben sich die Beobachtermatrizen $\boldsymbol{\Gamma}_{\mathrm{opt}}=\boldsymbol{I}_{2}, \boldsymbol{L}_{p, \mathrm{opt}}=\mathbf{0}, \boldsymbol{V}_{1, \mathrm{opt}}=\mathbf{0}$ und

$$
\begin{aligned}
& \boldsymbol{V}_{2, \mathrm{opt}}=\left[\begin{array}{cc}
-1,334 & 11,940 \\
-19,617 & 15,314
\end{array}\right], \\
& \boldsymbol{\Phi}_{\mathrm{opt}}=\left[\begin{array}{rr}
-5,634 & 1,757 \\
1,896 & -4,806
\end{array}\right], \quad \boldsymbol{L}_{i, \mathrm{opt}}=\left[\begin{array}{ll}
-4,452 & 3,862 \\
-9,801 & 7,897
\end{array}\right] .
\end{aligned}
$$

Anhand von Bild 4a und b wird deutlich, dass der optimierte Beobachter eine höhere Detektionsgeschwindigkeit erreicht und dabei die vorgegebene maximale Störverstärkung einhält.

\section{Zusammenfassung}

In diesem Beitrag wurde eine neue Entwurfsmethode für dynamische Entkopplungsregler vorgestellt. Mit Hilfe von Dualitätsbetrachtungen lässt sich mit dieser Methodik ein Verfahren zum Entwurf dynamischer Fehlerisolationsbeobachter ableiten, welches zusätzliche Freiheitsgrade gegenüber statischen FIOs bietet. Es wurde gezeigt, wie sich diese zur verbesserten 
Unterdrückung hochfrequenter Störungen sowie zur Steigerung der Detektionsgeschwindigkeit nutzen lassen. Andere Anwendungen der erweiterten Dynamik in den Diagonalpfaden sind Gegenstand laufender Forschungsarbeiten (s. z. B. [31]).

\section{Danksagung}

Diese Arbeit wurde unterstützt von der Deutsche Telekom Stiftung (http://www.telekom-stiftung.de).

\section{Literatur}

[1] Chen, B.; Nagarajaiah, S.: Linear-Matrix-Inequality-Based Robust Fault Detection and Isolation Using the Eigenstructure Assignment Method. In: Journal of Guidance, Control and Dynamics 30 (2007), Nr. 6, S. 1831-1835.

[2] Chen, J.; Patton, R. J.: Robust Model-Based Fault Diagnosis for Dynamic Systems. Kluwer Academic Publishers, 1999.

[3] Cremer, M.: A precompensator of minimal order for decoupling a linear multi-variable system. In: International Journal of Control 14 (1971), Nr. 6, S. 1089-1103.

[4] Cremer, M.: Festlegen der Pole und Nullstellen bei der Synthese linearer entkoppelter Mehrgrößenregelkreise, Teil 1. In: Regelungstechnik und Prozeß-Datenverarbeitung 21 (1973), Nr. 5, S. $144-150$

[5] Cremer, M.: Festlegen der Pole und Nullstellen bei der Synthese linearer entkoppelter Mehrgrößenregelkreise, Teil 2. In: Regelungstechnik und Prozeß-Datenverarbeitung 21 (1973), Nr. 5, S. 195-199.

[6] Cui, Y.; Huang, X.-H.; Wang, M.: Multi-objective Robust Fault Detection Filter Design in a Finite Frequency Range. In: Proceedings of the 6th International Symposium on Neural Networks: Advances in Neural Networks - Part III, 2009, S. 733-743.

[7] Dai, X.; GAO, Z.; Breikin, T.; Wang, H.: Zero Assignment for Robust $H_{2} / H_{\infty}$ Fault Detection Filter Design. In: IEEE Transactions on Signal Processing 57 (2009), Nr. 4, S. 1363-1372.

[8] Davoodi, M. R.; Talebi, H. A.; Momeni, H.: A Novel Simultaneous Fault Detection and Control Approach Based on Dynamic Observer. In: Proceedings of the 18th IFAC World Congress, 2011, S. 12036-12041.

[9] Ding, S.X.: Model-based Fault Diagnosis Techniques. Springer, 2008.

[10] Falb, P. L.; Wolovich, W. A.: Decoupling in the Design and Synthesis of Multivariable Control Systems. In: IEEE Transactions on Automatic Control 12 (1967), Nr. 6, S. 651-659.

[11] Frank, P. M.: Fault Diagnosis in Dynamic Systems Using Analytical and Knowledge-based Redundancy - A Survey and Some New Results. In: Automatica 26 (1990), Nr. 3, S. 459-474.

[12] Herrera H., A. N.; Lafay, J. F.: New results about Morgan's problem. In: IEEE Transactions on Automatic Control 38 (1993), Nr. 12, S. 1834-1838.

[13] Istermann, R.: Fault-Diagnosis Systems. Springer, 2006.

[14] IWASAKI, T.; HARA, S.: Generalized KYP lemma: unified frequency domain inequalities with design applications. In: IEEE Transactions on Automatic Control 50 (2005), Nr. 1, S. 41-59.

[15] Jaimoukha, I. M.; Li, Z.; MazArs, E.: Fault isolation filter with linear matrix inequality solution to optimal decoupling. In: Proceedings of the American Control Conference, 2006, S. 2339-2344.

[16] Li, Z.; Mazars, E.; Zhang, Z.; Jaimoukha, I. M.: State-space solution to the $H_{-} / H_{\infty}$ fault-detection problem. In: International Journal of Robust and Nonlinear Control (2011).

[17] Li, Z.; Jaimoukha, I. M.: Observer-based fault detection and isolation design for linear time-invariant systems. In: International Journal of Control 82 (2009), Nr. 1, S. 171-182.
[18] LiU, B.; SI, J.: Fault isolation filter design for linear time-invariant systems. In: IEEE Transactions on Automatic Control 42 (1997), Nr. 5, S. 704-707.

[19] Lohmann, B.: Vollständige Entkopplung durch dynamische Zustandsrückführung. In: at - Automatisierungstechnik 39 (1991), Nr. 9, S. 459-464.

[20] LöFBERG, J.: YALMIP: a toolbox for modeling and optimization in MATLAB. In: Proceedings of the IEEE International Symposium on Computer Aided Control Systems Design, 2004, S. 284-289.

[21] Mazars, E.; Jaimoukha, I. M.; Li, Z.; Zolotas, A. C.: Fault detection and isolation filter design for systems subject to polytopic uncertainties. In: Proceedings of the Mediterranean Conference on Control and Automation, 2007, S. 1-5.

[22] Moness, M.; Amin, M.: Minimal-order precompensators for decoupling linear multivariable systems (A,B,C,E). In: International Journal of Control 47 (1988), Nr. 6, S. 1925-1936.

[23] Park, J.; Rizzoni, G.; Ribbens, W. B.: On the Representation of Sensor Faults in Fault Detection Filters. In: Automatica 30 (1994), Nr. 11, S. 1793-1795.

[24] Park, J.-K.; Shin, D.-R.; Chung, T. M.: Dynamic observers for linear time-invariant systems. In: Automatica 38 (2002), Nr. 6, S. $1083-1087$.

[25] Pertew, A. M.; Marquez, H. J.; Zhao, Q.: $H_{\infty}$ Dynamic observer design with application in fault diagnosis. In: Proceedings of the IEEE Conference on Decision and Control, 2005, S. 3803-3808.

[26] Pertew, A. M.; Marquez, H. J.; Zhao, Q.: LMI-based sensor fault diagnosis for nonlinear Lipschitz systems. In: Automatica 43 (2007), Nr. 8, S. 1464-1469.

[27] Roppenecker, G.; Lohmann, B.: Vollständige modale Synthese von Entkopplungsregelungen. In: at - Automatisierungstechnik 36 (1988), Nr. 11, S. 434-441.

[28] Stefanovski, J.: Sufficient conditions for linear control system decoupling by static state feedback. In: IEEE Transactions on Automatic Control 46 (2001), Nr. 6, S. 984-990.

[29] SVARICeK, F.: Zuverlässige numerische Analyse linearer Regelungssysteme. Vieweg \& Teubner, 1995.

[30] Toн, K. C.; Todd, M.J.; TÜTÜNCÜ, R. H.: SDPT3 - A Matlab software package for semidefinite programming. In: Optimization Methods \& Software 11 (1999), Nr. 1-4, S. 545-581.

[31] Wahrburg, A.; Adamy, J.: Observer-based fault isolation for statically non-isolable linear systems. In: IEEE Multi Conference on Systems and Control, 2012, - akzeptiert.

[32] WANG, H.; YANG, G.-H.: Fault Estimations for Uncertain Linear Discrete-time Systems in Low Frequency Domain. In: Proceedings of the American Control Conference. New York City, 2007, S. 1124-1129.

[33] WANG, H.; YANG, G.-H.: A finite frequency approach to filter design for uncertain discrete-time systems. In: International Journal of Adaptive Control and Signal Processing 22 (2008), Nr. 6.

[34] WANG, J. L.; YANG, G.-H.; LiU, J.: An LMI approach to $H_{-}$index and mixed $H_{-} / H_{\infty}$ fault detection observer design. In: Automatica 43 (2007), Nr. 9, S. 1656-1665.

[35] Wei, M.; WAng, Q.; Cheng, X.: Some new results for system decoupling and pole assignment problems. In: Automatica 46 (2010), Nr. 5, S. 937-944.

[36] WeI, X.; LinUA, L.; CAI, G.; QIN, Y.; JiA, L.: Robust fault detection dynamic observer design for LTI systems. In: Proceedings of the Chinese Control and Decision Conference, 2010, S. 1229-1234.

[37] Zhong, M.; Ding, S. X.; LAM, J.; WAng, H.: An LMI approach to design robust fault detection filter for uncertain LTI systems. In: Automatica 39 (2003), Nr. 3, S. 543-550. 


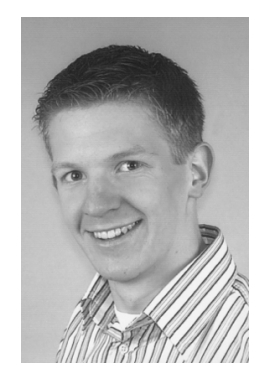

Dipl.-Ing. Arne Wahrburg ist wissenschaftlicher Mitarbeiter am Fachgebiet Regelungstheorie und Robotik im Institut für Automatisierungstechnik der Technischen Universität Darmstadt. Hauptarbeitsgebiete: Modellbasierte Fehlerdiagnose, Entkopplungsregelungen, Verteilte Systeme.

Adresse: siehe unten,

E-Mail: wahrburg@rtr.tu-darmstadt.de

Verfügbar unter

lediglich die vom Gesetz vorgesehenen Nutzungsrechte gemäß UrhG

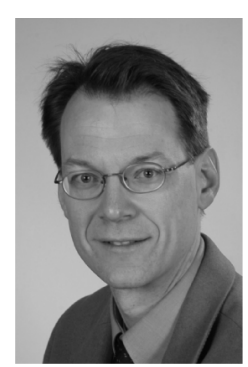

Prof. Dr.-Ing. Jürgen Adamy ist Leiter des Fachgebiets Regelungstheorie und Robotik im Institut für Automatisierungstechnik der Technischen Universität Darmstadt. Hauptarbeitsgebiete: Regelungsverfahren, Computational Intelligence, autonome mobile Roboter.

Adresse: Technische Universität Darmstadt, Institut für Automatisierungstechnik, Fachgebiet Regelungstheorie und Robotik, Landgraf-GeorgStr. 4, D-64283 Darmstadt, Tel: +49-(0)6151-163442, E-Mail: jadamy@rtr.tu-darmstadt.de

\section{Vorschau auf Heft 9/2012}

Unsere nächste Ausgabe ist ein Schwerpunktheft zur GMA 1.3 (Gastherausgeber: A. Sawodny) und enthält unter anderen folgende Beiträge:

- Buchholz, M.: Verbesserte Subspace-Identification für lineare Systeme mit variierenden Parametern durch rekursive Schätzung

- Gehring, N.: Algebraische Methoden zur Parameteridentifikation für das schwere Seil

- Harkort, Ch.: Strukturerhaltende Approximation linearer verteilt-parametrischer PCHD-Systeme

- Lein, Cl.: MORPACK-Schnittstelle zum Import von FE-Strukturen nach SIMPACK

Weitere Informationen über geplante Hefte, ausführliche Informationen über die in den letzten Heften der at erschienenen Beiträge sowie Hinweise für Autoren finden Sie im Internet unter http://www.at-automatisierungstechnik.de. 